\title{
Sub-Ångstrom EDX Mapping Enabled by a High-brightness Cold Field Emission Source
}

Berit Goodge ${ }^{1}$, Anna Carlsson², Maarten Bischoff ${ }^{2}$, Ali Mohammadi-Gheidari², Gamal Fallag ${ }^{2}$, Greg Schwind $^{3}$, Christian Maunders ${ }^{2}$, David Muller ${ }^{1}$ and Lena Kourkoutis ${ }^{1}$

${ }^{1}$ Cornell University, Ithaca, New York, United States, ${ }^{2}$ Thermo Fisher Scientific, Eindhoven, NoordBrabant, Netherlands, ${ }^{3}$ BeamTech, Hillsboro, Oregon, United States

Recent years have seen a surge in the push for precise chemical and structural analysis in the (S)TEM at high spatial resolution. Ranging from atomic-resolution elemental mapping [1] to picometer-precision atom tracking [2], these efforts reflect the growing importance of quantitative measurements at the nanoscale across a wide variety of fields, including quantum materials [3], energy systems [4], and biological sciences [5]. For many of these systems, understanding subtle variations in elemental distribution down to the atomic scale is of the utmost importance. While atomic resolution EDX mapping has been previously demonstrated, results have thus far been limited by tradeoffs between probe current and convergence angle [6], especially in attempts to map the lighter elements. Recently, Thermo Fisher Scientific developed a high brightness $30-300 \mathrm{kV}$ capable cold field emission gun (X-CFEG) electron source. The high coherent probe current enables sub- $\AA$ resolution atomic mapping by EDX and retains deep sub-Å resolution in HAADF STEM imaging with over 500 pA of beam current.

Figure 1 demonstrates atomic-resolution elemental mapping of a $\mathrm{DyScO}_{3}$ crystal by STEM-EDX at 200 $\mathrm{kV}$ in a Spectra 300 equipped with an X-CFEG, a S-CORR corrector, and a 1.8 steradian Dual-X EDX detector. The data shown here were recorded at 256x256 px and $10 \mu \mathrm{s} / \mathrm{px}$ dwell time using a $25 \mathrm{mrad}$ convergence angle and a probe current of $\sim 120 \mathrm{pA}$. With these rapid acquisitions, nearly 2000 consecutive frames are registered and summed to produce high signal-to-noise ratio (SNR) elemental maps. Inset fast Fourier transforms (FFTs) of each map are shown, demonstrating sub- $\AA$ information transfer in the higher SNR Dy and Sc maps, and near- $\AA$ information transfer in the lower SNR O map. The "twisted dumbbells" of Dy, characteristic of this (001) orthorhombic projection (Fig. 1i), are also clearly resolved (Fig. 1a, d). The composite RGB map of Dy, Sc and O (Fig. 1f) illustrates direct elemental mapping at the atomic scale with light-element resolution approaching that of more indirect contrast techniques, such as annular bright-field (ABF) STEM. Compared to ABF STEM, EDX mapping has the added benefit of resolving the crystalline sub-structures by atomic species. Here, the FFTs of each elemental map highlight the different crystalline symmetries of the respective sublattice, particularly noticeable in the pseudocubic arrangement of Sc as compared to the face-centered $\mathrm{O}$ sites.

Beyond structural information, the same data set also demonstrates high sensitivity to elemental concentrations, as evidenced by the substitutional point defects of Dy onto Sc sites (Fig. 1d-e). Line profiles of the Dy and Sc elemental maps across a single row of atoms confirm a visually apparent substitution with a distinct peak in the Dy intensity and a corresponding decrease in the Sc intensity at the same location.

Such high-resolution elemental STEM-EDX mapping is enabled in large part by the X-CFEG's ability to concentrate very high currents into a sub- $\AA$ probe. Figure 2 shows a direct demonstration of this capability, as evidenced by the deep sub- $\AA$ resolution achieved with a probe current exceeding $500 \mathrm{pA}$ at $300 \mathrm{kV}$. HAADF STEM imaging with a relatively standard probe current of $80 \mathrm{pA}$ (Fig. 2a) shows information transfer out to $\sim 0.61 \AA$ (Fig. $2 b)$ in a single frame $(2048 \times 2048 \mathrm{px}, 0.25 \mu \mathrm{s} / \mathrm{px}$ dwell time). The (001) orthorhombic projection of $\mathrm{DyScO}_{3}$ shown here (the same as in Fig. 1i) results in a $\sim 0.7 \AA$ projection separation between alternating stacked Dy atoms which can be clearly resolved. Cross-correlation and 
rigid registration [7] of several image frames (Fig. 2c) further improves the achievable resolution, reaching information transfer near $0.56 \AA$ (Fig. 2d). Increasing the probe current to more than 500 pA (Fig. 2e) allows a reduction in scan pixel dwell time to only $50 \mathrm{~ns} / \mathrm{px}$ while preserving atomic-resolution. Registration of several images frames again significantly improves resolution, demonstrating information transfer at $0.66 \AA$ and recovering the distinction between Dy dumbbell atoms.

It is interesting to note that given the rapid scan frame rates used for very high current imaging, the total exposed electron doses for the registered images in Fig. $2 \mathrm{c}$ and $\mathrm{g}$ are nominally not very different. While the probe current in Fig. 2e-g is approximately 7 times higher than the more standard current used in Fig. 2a-d, the per-pixel dwell time of the scan is also 5 times faster. The potential for such rapid frame-rate STEM (i.e., over 70 frames/second at $512 \times 512$ pixels) extends to not only high-throughput experiments, but also to novel observation of dynamic in situ processes without sacrificing the quantitative advantages of atomic resolution HAADF STEM. [8]
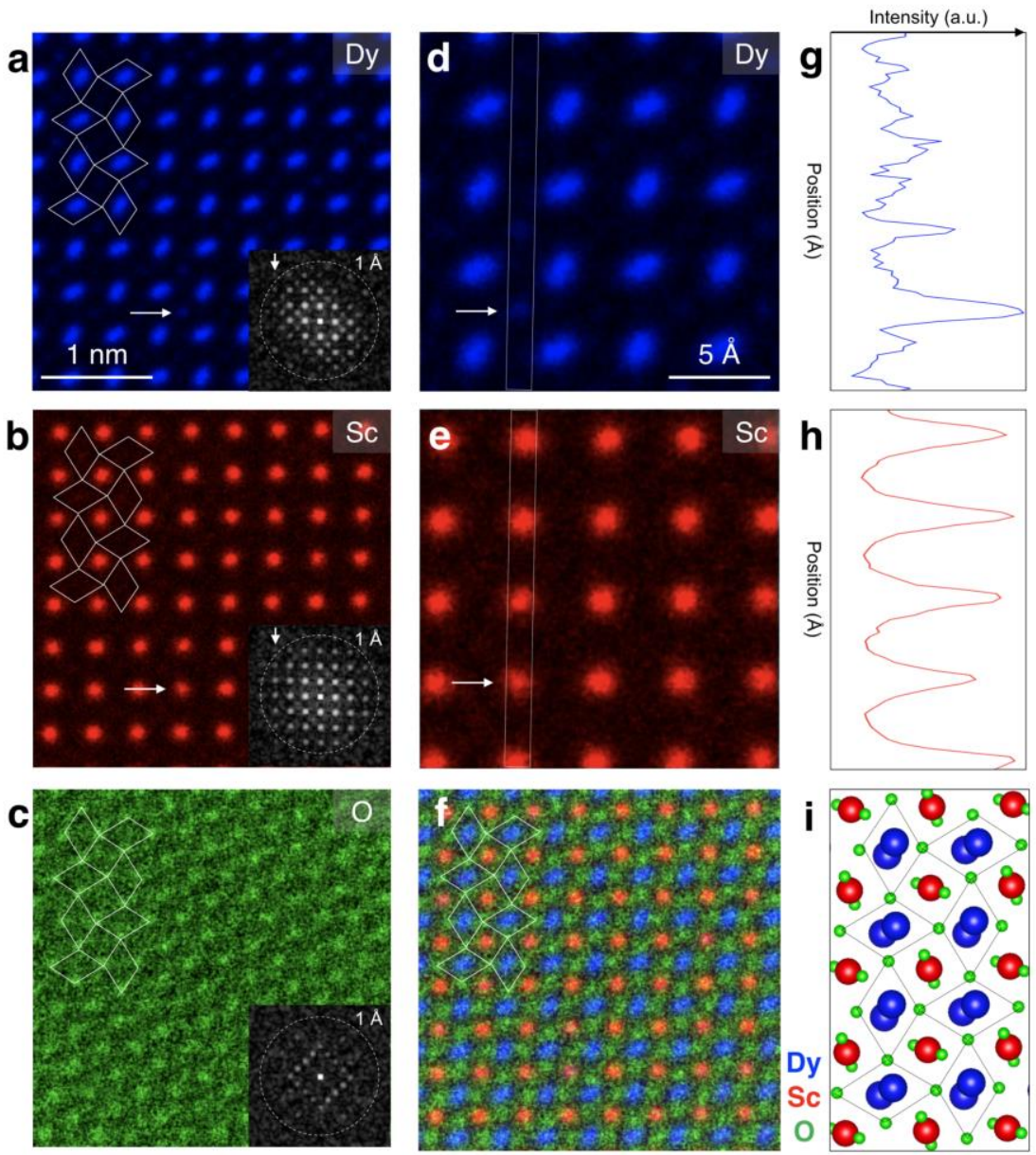

Figure 1. Sub- $\AA$ STEM-EDX elemental mapping of DyScO3 using a $200 \mathrm{kV}$ Spectra 300 with cold field emission source and Dual-X EDX detector. The distorted perovskite structure is directly visible in the [001] orthorhombic projection (i), especially as "twisting dumbbells" of Dy and rotations of the $\mathrm{O}$ octahedra (a-c, f). Inset FFTs for each map demonstrate sub- $\AA$ information transfer in Dy and Sc and highlight the different periodic symmetries of each elemental species. Additionally, the SNR of the EDX map is sufficient to resolve a substitutional point defect of Dy onto a Sc site, marked by horizontal white 
arrows, and confirmed as an increase (decrease) in the intensity profiles of the Dy (Sc) maps across the boxed regions (d-e, g-h).

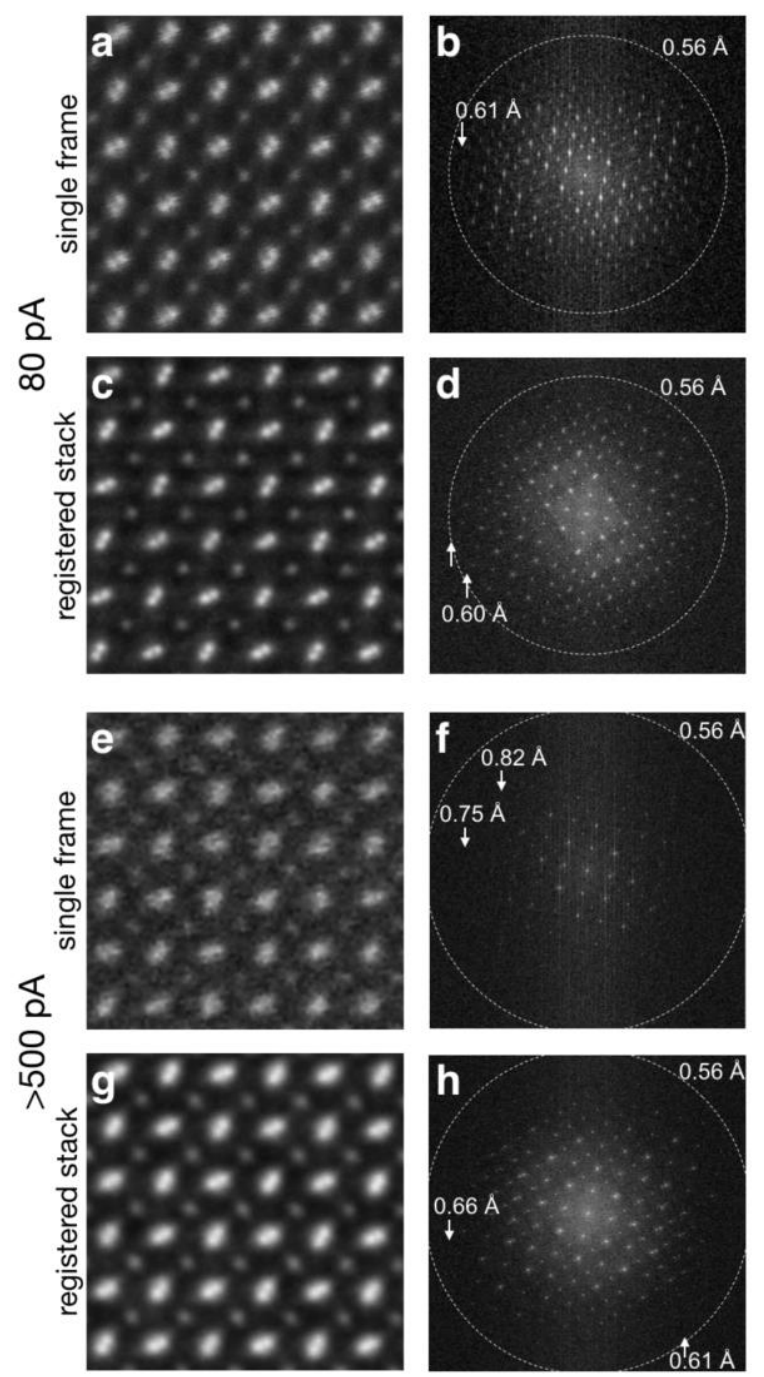

Figure 2. HAADF STEM images of the distorted perovskite DyScO3 (same structure as in Fig. 1i) show deep sub- $\AA$ information transfer with high beam currents in the $300 \mathrm{kV}$ Spectra $300 \mathrm{X}-\mathrm{CFEG}$. A singleframe scan $(2048$ x $2048 \mathrm{px}, 0.25 \mu \mathrm{s} / \mathrm{px})$ with $80 \mathrm{pA}$ probe current is sufficient to resolve the $\sim 0.7 \AA$ projection spacing between atoms in the Dy dumbbells (a-b). The SNR and resolution can both be improved with rigid registration of several frames (c-d). With more than $500 \mathrm{pA}$ of beam current, the dwell time can be reduced to $50 \mathrm{~ns} / \mathrm{px}$ while still preserving atomic-resolution (e-f). Atomic separation in the Dy dumbbells can be recovered in a registered stack of several rapid scan frames.

\section{References}

[1] Muller, et al. Science 319 (2008), p. 1073-1075.

[2] Yankovich, et al. Nature Communications 5 (2014), p. 4155.

[3] El Baggari, et al. Proceedings of the National Academy of Sciences 115 (2018), p. 1445-1450.

[4] Zachman, et al. Nature 560 (2018), p. 345-349.

[5] Smeets, et al. Microscopy and Microanalysis 25 S2 (2019), p. 1722-1723.

[6] Chen, et al. Ultramicroscopy 168 (2016), p. 7-16. 
[7] Savitzky, et al. Ultramicroscopy 191 (2018), p. 56-65.

[8] Supported by PARADIM, an NSF-MIP (DMR-1539918), with additional support by NSF (DMR1429155, DMR-1719875). DyScO 3 sample provided by D. G. Schlom (Cornell University). 